\title{
Chapter 18. Antihypertensive therapy
}

(C) Japanese Society of Nephrology 2009

- Significance of blood pressure control in CKD resides in suppression of CKD progression and of CVD development as well as CVD progression.

- The target blood pressure is less than 130/80 $\mathrm{mmHg}$.

- Home monitoring of blood pressure is important. Blood pressure is gradually reduced.

- In blood pressure control, modification of lifestyle and salt restriction are important.

- In principle, ACE inhibitors or ARBs is chosen as first-line antihypertensive agent.

- Combination therapy is necessary to achieve target blood pressure in the majority of cases.

- It is better to reduce urinary protein excretion below $0.5 \mathrm{~g} / \mathrm{g}$ creatinine.

The importance of decreasing blood pressure in CKD

- Hypertension is a cause of CKD and aggravates existing CKD. On the contrary, CKD brings about hypertension and worsens existing hypertension. A vicious cycle thus arises between the two illnesses.

- The purpose of blood pressure control is to suppress CKD progression and to prevent or retard the progression to ESKD.

- Suppression of CKD progression leads to inhibition of development as well as progression of cardiovascular disease (CVD).

- Hypertension is a potent risk factor for CVD, so that antihypertensive therapy contributes directly to CVD development as well as its progression.

\section{Target blood pressure in CKD}

- Meta-analysis revealed that greater blood pressure reduction results in smaller GFR decline rate (Fig. 18-1).

- The target blood pressure in CKD is set at less than $130 / 80 \mathrm{mmHg}$, and if urinary protein exceeds $1 \mathrm{~g} /$ day it is set further lower at $125 / 75 \mathrm{mmHg}$.

\section{Importance of home blood pressure monitoring}

- Home blood pressure monitoring is essential to detect nocturnal and morning hypertension, which are risk factors for progression of CKD.

- CKD patients are required to measure blood pressure twice a day: (1) within $1 \mathrm{~h}$ of waking up in the morning, before breakfast and (2) before going to bed at night. Physicians make use of both home and office blood pressure, which is useful for management of hypertension.

\section{Speed of blood pressure lowering}

- Strict blood pressure control is essential for CKD but its rapid attainment has potential to aggravate kidney function and CVD.

- Blood pressure is gradually decreased in 2-3 months under close observation. 
Table 18-1 Lifestyle modifications

\begin{tabular}{ll}
\hline 1. & Restriction of salt intake to less than $6 \mathrm{~g} / \mathrm{day}$ \\
2. & $\begin{array}{l}\text { Increased intake of vegetables and fruits } \\
\text { a }\end{array}$ \\
3. & Maintenance of appropriate body weight: not exceeding BMI ([body weight $\left.(\mathrm{kg})] /[\mathrm{height}(\mathrm{m})]^{2}\right)$ of 25 \\
4. & Exercise: indicated for hypertensive patients without cardiovascular disease \\
5. & Regular aerobic exercise for 30 min or longer every day \\
6. & No smoking
\end{tabular}

Comprehensive modification of one's lifestyle is more effective

Quoted from: Lifestyle Modifications in Japanese Society of Hypertension Guidelines for the Management of Hypertension (JSH 2004). Hypertens Res 2006;29(Suppl):S1-S105

${ }^{a}$ Increased intake of vegetables and fruits is not recommended in patients with severe renal dysfunction, because it may induce hyperkalemia. Also, increased intake of fruits is not recommended in diabetic patients, because it may lead to an increase in calories

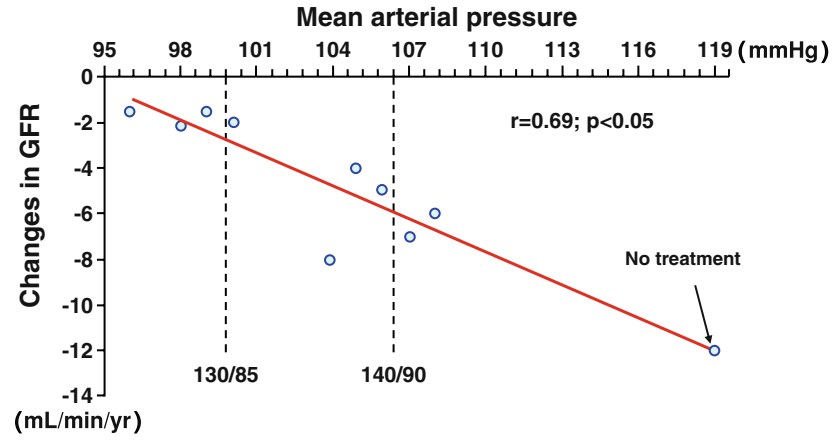

Fig. 18-1 Relationship between achieved blood pressure control and declines in GFR in clinical trials of diabetic and nondiabetic renal disease. Quoted, with modification, from: Bakris et al. Am J Kidney Dis 2000;36:646-661

\section{Lifestyle modification and salt restriction}

- Lifestyle modification is essential for management of hypertension (Table 18-1).

- Salt restriction is particularly essential. Physicians should advise patients to take less than $6 \mathrm{~g} /$ day salt. Salt restriction enhances antihypertensive effects of ACE inhibitors and ARBs.

- In the elderly, excessive salt restriction may disturb appetite, resulting in dehydration, leading to reduced kidney function.

- When salt restriction is difficult, a small dose of diuretics may be useful in combination. Concurrent use of thiazide diuretics (CKD stages 1-3) or loop diuretics (CKD stages 3-5) can accelerate salt excretion. However, physicians are to be aware of possible complications of diuretics such as hypokalemia, hyperuricemia, and dehydration.

\section{Kidney protection by ACE inhibitors or ARBs}

- Kidney protection by ACE inhibitors and ARBs has been demonstrated.

- These agents are recommended for diabetic nephropathy with hypertension and even without hypertension.

- Nondiabetic CKD patients are expected to benefit from ACE inhibitors and ARBs. These agents, therefore, are prescribed if blood pressure is high.

\section{Caution for administration of ACE inhibitors or ARBs}

- Administration of ACE inhibitors or ARBs may increase serum creatinine level. Despite this, these agents are allowed to be continued, placing priority on pharmacological effects unless an increment of serum creatinine exceeds $30 \%$ of previous level or $1 \mathrm{mg} / \mathrm{dL}$. For example, these agents may be continued if serum creatinine is elevated from 1.34 to $1.74 \mathrm{mg} / \mathrm{dL}$ after starting treatment. Serum creatinine and potassium are measured at 2 weeks or 1 month after starting ACE inhibitors or ARBs, and if continued, they are constantly monitored thereafter.

- If serum creatinine is elevated to the above-mentioned degree, these agents should be reduced in dosage or discontinued, and consultation to nephrologists is required. The same is true of elevated serum potassium level more than $5.5 \mathrm{mEq} / \mathrm{L}$.

- Serum creatinine level may be excessively elevated due to: (1) renal artery stenosis, (2) administration of NSAIDs, (3) heart failure, (4) dehydration or (5) urinary tract abnormality. If these are possible, ACE inhibitors or ARBs is carefully continued or should be discontinued. 
- Physicians are always aware that elderly patients can easily fall into dehydration in summertime and that NSAIDs are frequently prescribed by other medical providers, which may injure kidney.

\section{Combination therapy to achieve target blood pressure}

- In clinical studies, 3-5 antihypertensive agents are usually used in combination for strict blood pressure control.

- Other agents are combined when monotherapy by ACE inhibitors or ARBs fails to achieve the target blood pressure.

Diuretics

- A combination of a diuretic in a small dose can enhance antihypertensive effects of other agents.

Calcium-channel blocking agents (CCBs)

- CCBs, if combined with other agents, strictly lower blood pressure and suppress CKD progression more easily.

Other antihypertensive agents

- There is no clinical evidence of $\alpha$-blockers, $\beta$-blockers or central sympatholytic agents being effective directly in
CKD. These agents however are expected to suppress CKD progression through lowering blood pressure.

\section{Prevention of decline in GFR through reduction of urinary protein excretion}

- Urinary protein is a critical risk factor for progression of CKD. It is considered that prognosis of CKD can be prevented by reduction of urinary protein.

- ACE inhibitors and ARBs are superior to other antihypertensive agents in reducing urinary protein. Beneficial effects of these drugs on CKD progression depend mainly on their decreasing effects on urinary protein.

- If sufficient reduction of urinary protein is not attained, it is recommended that ACE inhibitors or ARBs be increased in dose to maximum while attention is being paid to blood pressure and adverse effects.

- ACE inhibitors or ARBs are demonstrated to reduce CVD events through alleviating microalbuminuria or proteinuria.

- The target of urinary protein reduction is less than $0.5 \mathrm{~g} / \mathrm{g}$ creatinine. 